\title{
XAS of Ruthenium Phthalocyanine Dimer: Structural Properties of Bulk and Film Form
}

\author{
L. Alagna, A. Capobianchi, G. Pennesi, T. Prosperi and G. Rossi \\ ICMAT-CNR Area della Ricerca di ROMA, P.O. Box 10, 00016 Monterotondo, Italy
}

\begin{abstract}
We measured in absorption mode at the Ruthenium K-edge, Ru-Ru and Ru-N distances, in structurally known systems: the Ruthenium dimeric precursor compound, already structurally characterized, the monomeric species of Ruthenium phthalocyanine (adduct) and the polymeric species of Ruthenium phthalocyanine (with bridging oxygen). The film, a candidate for sensor and electrochromic applications, is loaded by sublimation and has been measured in fluorescence straight on the growth substrate (glass) obtaining excellent data.

In the Fourier transforms obtained for the dimer, a Ru-Ru bond is well defined. For the monomer no Ru-Ru distance is present within the fit of the first neighbours while the polymeric species shows a well defined peak, at ca. $3.38 \AA$, due to the moiety Ru-O-Ru, absent in the other two cases. In the film case no evidence is present for a Ru-O-Ru bond, while it is evident that a Ru-Ru bond at $2.35 \AA$ is present at the same distance as in the dimer. Good fit of the film data are indeed obtained by imposing the dimer parameters in perfect agreement within the first two shells.
\end{abstract}

\section{INTRODUCTION}

The phthalocyanines are a special class of porphyrin (1) like molecules where a different metal ion and the substitution of the ligands axially to the flat phthalocyanine plane, leads to very interesting behaviour as catalysts and electrocatalysts, sensors, displays, devices and information storage systems. In our case the systems under study are the dimeric Ruthenium phthalocyanine and the final product obtained after reaction with molecular oxygen. The Ruthenium derivatives are a very promising with respect to electronic properties, which role it not yet widely investigated, for structure and behaviour. An exhaustive work had been already carried out in preparation and characterization of the Ru compounds $(2,3)$, showing that Ruthenium phthalocyanine is an amorphous material consisting of dimeric molecules stacked parallel to the axis of intradimer $\mathrm{Ru}-\mathrm{Ru}$ bond direction with a short range order. The magnetic behaviour and the electrical conductivity have been studied and related to structural information obtained by LAXS (Large-Angle X-ray Scattering) data. Furthermore the interaction of Ruthenium phthalocyanine with dioxygen in tetrahydrofuran and his oxidative catalysis by $\mathrm{O}_{2}$ have been investigated. Despite the unexplored potentiality of the system no structural information are up to now available for the film, due to the amorphous state of the compound.

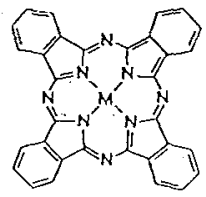

Figure 1 : Schematic drawing of one planar unit of phthalocyanine (monomer case). The Ruthenium is seated in the center with bond lenghts forced from the rings geometry.

\section{EXPERIMENTAL}

We measured at GILDA CRG beamline, ESRF, at the Ruthenium K-edge (21126 eV), absorption EXAFS of Ru-Ru and Ru$\mathrm{N}$, structurally characterized systems: 1-Monomeric Ru-Phthalocyanine (bis-pyridine adduct); 2-polymeric Ru- Phthalocyanine (oxo-bridged); 3-Dimeric Ru-Phthalocyanine. The fluorescence spectrum at L.N. temperature has been recorded for the film deposited by sublimation of the dimeric starting material, on a cold (glass) substrate.

Data analysis has been performed using standard methods. EXCURVE has been used to fit the data, describing the highly symmetric four pyrrolic rings around the metal ion up to $\beta-C$ and taking into account the multiple scattering effects, heavily 
contributing in such systems. Metallic Ruthenium and dimeric derivative had been used as standards.

\section{RESULTS AND DISCUSSION}

In figure 2 ( $a$ and $b$ ) is reported the comparison between the Fourier transforms of the various Ruthenium derivatives where differences are evident both in peak position and amplitude. Important differences are present in the axial bonds to the metal ion and in the second shell contributions due, with exception of the monomer, to the presence of a second Ruthenium atom. In the case of the Monomeric Ruthenium Phthalocyanine (bis-pyridine adduct) no Ru-Ru contribution is present, but a long axial Ru$\mathrm{N}$ at $2.538 \AA$, belonging to the two axial ligands appears, while for the polymer, where the units are axially stacked together throught a short Ru-O bond at $1.807 \AA$, a second unit is linked at a distance of $3.719 \AA$. In the case of the dimer the Ru-Ru contribution appears at $2.384 \AA$, and the second unit is seen at $4.417 \AA$. All the Ru-Ru distances are consistent with EXAFS data for similar systems [4] and with the available LAXS data $[2,3]$ for the derivatives under study within $0.02 \AA$.
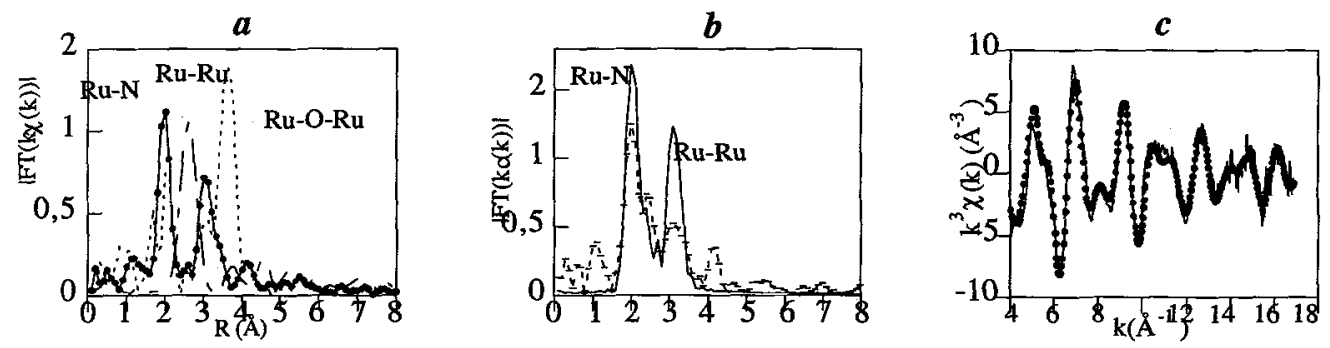

Figure 2. $a$ : Metallic Ruthenium( -.-.)polymer(...) and Monomer (-) FT modulus; $b$ : Dimer( -.-.) and Film (-) ; $c:$ Monomer experimental extracted EXAFS and best fit .

To correctly assign all the features, the complete fit analysis has been undertaken for all the compounds taking into account all the relevant contributions to the signal, but constrained by the presence of four fixed pyrrolic rings in the molecular plane, with high geometry. In figure $2 c$ is reported the result of the fit for the basic monomeric Ruthenium Phthalocyanine (see TABLE for results). Analogous fits for the other compounds gave the results reported in the TABLE. In the case of the film the fit of the first two shells clearly indicated a structure similar to the Dimeric Phthalocyanine. No axial ligands are indeed contributing to the first shell, while one Ruthenium atom is present at the same distance as found in the dimeric derivative and therefore consistent with a Ru-Ru bond.

TABLE. Interatomic distances in $\AA$ from EXCURVE constrained refinement including up to $\beta-\mathrm{C}$ and multiple scattering effects. R-factor obtained are between $30 \%$ (Monomer) and $48 \%$ over an interval of $1300 \mathrm{eV}$ (polymer) for non-Fourier filtered data.

$\begin{array}{lcccccc}\text { Samples } & \text { Ru-N (planar) } & \text { Ru-X (axial) } & \text { Ru-Ru (1st) } & \text { Ru-Ru (2nd) } & \text { Ru-C- } \beta \text { (ring) } & \text { Ru-N pl 450 } \\ \text { Ru Metal } & & & 2.70 & 3.38 & & \\ \text { Ru Monomer } & 2.00 & 2.54(\mathrm{X}=\mathrm{N}) & & & 3.01 & 3.32 \\ \text { Ru polymer } & 1.99 & 1.81(\mathrm{X}=\mathrm{O}) & 3.72 & & 2.98 & 3.33 \\ \text { Ru Dimer } & 1.98 & & 2.38 & 4.42 & 3.01 & 3.26 \\ \text { Ru Film } & 2.01 & & 2.35 & & 3.05 & 3.25\end{array}$

The available data clearly describe the first two shells of the film, which is produced using the dimer as starting material: at the present we can already conclude that the structure of the dimer is retained, at least for the first surroundings, in the film. At this point it had been possible to precisely determine the structure of Ru-phthalocyanine dineric species, once loaded as amorphous film on a substrate. The good quality data allowed a good quality fit and led to the first structural characterization of the film as a material of technological interest. The present results are useful to understand the spectroscopic evidences of the film, and to explain its behaviour, both in terms of chemical reactivity towards small molecules (chemical sensors) as in the oxo-reductions properties (electrochromic devices). Further investigations will be carried to improve the efficiency of the system, that is already very promising in the field of chemical engineering.

\section{References}

[1]D.Wöhrle"Phthalocyanines",C.C.Leznoff and A.B.P.Lever Eds.,VCHPublisher,1989,133;A.W. Snow,W.R.Barger ibidem 341 [2]Capobianchi A., Paoletti A.M., Pennesi G., Rossi G., Caminiti R., Ercolani C., Inorg. Chem. 33, (1994), 4635.

[3]Capobianchi A., Pennesi G., Paoletti A.M., Rossi G., Caminiti R., Sadun, C. Ercolani C., Inorg. Chem. 33, (1996), in press. [4]Asahina H., Zisk M.B.,Hedman B.,McDevitt T.M., Coliman J.P., Hodgson K.O., J. Chem. Soc. Chem. Comm., 1360, (1989). 